\title{
Phenytoin-Induced Nephrotic Syndrome
}

\begin{tabular}{|l|l|l|}
\hline G. & Guido & Orlandini \\
\hline G. & Giovanni & Garini \\
\hline
\end{tabular}

Institute of Clinical Medicine and Nephrology, School of Medicine, Parma University, Parma, Italy

Guido Orlandini, Institute of Clinical Medicine and Nephrology, School of Medicine, Parma University, Via Gramsci 14, I43100 Parma (Italy)

Dear Sir,

An 83-year-old woman was admitted to our ward with nephrotic syndrome. Five years before, the patient had undergone surgery for a colonic adenocarcinoma (Duke classification stage B). 40 days before admission, therapy with phenytoin (100 $\mathrm{mg}$ u.i.d.) was started to control seizures (grand mal), occurring with increasing frequency, which were supposed to be caused by cerebrovascular disease. The patient appeared anasarcous. Massive proteinuria was present (about 10 $\mathrm{g}$ /day). Serum electrophoresis showed a polyclonal $\gamma$-peak (40.4\%). Complement fractions (C3 and $\mathrm{C} 4$ ) and the total hemo-lytic complement were within the normal range. All the possible causes of nephrotic syndrome were excluded step by step, although we did not perform a renal biopsy on account of the patient's age and clinical condition. However, the presence of some hyaline and granular casts without hematuria suggested minimal-change disease or membranous glomerulonephritis. 15 days after admission, we decided to withdraw phenytoin in relation to a single case report [1] of low-dosage phenytoin-in-duced nephrotic syndrome.

The clinical picture improved dramatically in a few days, and the serum electrophoretic pattern returned to normal. Proteinuria was still absent after 15,30 and 60 days. The chronic circulation of an antigen in low dosages (as in this case at a subtherapeutic concentration of $<$ IO $\mu \mathrm{mol} / \mathrm{l}$ ) could have induced the formation of immune complexes [2] or allowed their local formation in the subepithelial space [3]. Interactions of phenytoin with the immune response are also suggested by the evidence that the drug can modify the immunological alterations (although not the clinical course) occurring in IgA neph-ropathy [4].

We believe that the risk of developing nephrotic syndrome should be clearly indicated in the product information sheet in consideration of the therapeutic importance and widespread use of this drug.

References

Swainson CP, Thomson D, Short AIK, et al: Plasma exchange in the successful treatment of drug-induced renal disease. Neph-ron 1982;30:244-249.

Germuth FG, Rodriguez E: Immunopathology of the renal glomerulus. Boston, Little, Brown, 1973.

Izui S, Lambert PH, Miescher PA: In vitro demonstration of a particular affinity of glomerular basement membrane and collagen for DNA: a possible basis for a local formation of DNA-antiDNA complexes in SLE. J Exp Med 1976;144:428-443. Egido J, Rivera F, Sancho J, et al: Phenytoin in IgA nephropa-thy: a long-term controlled trial. Nephron 1984;38:30-39. 
